\title{
Acupuncture as an adjunct to exercise based physiotherapy for osteoarthritis of the knee: randomised controlled trial
}

\author{
Nadine E Foster, senior lecturer in therapies (pain management), ${ }^{1}$ Elaine Thomas, senior lecturer in \\ biostatistics, ${ }^{1}$ Panos Barlas, research fellow, ${ }^{2}$ Jonathan C Hill, arc lecturer in physiotherapy, ${ }^{1}$ \\ Julie Young, research nurse, ${ }^{1}$ Elizabeth Mason, research physiotherapist, ${ }^{1}$ Elaine M Hay, professor of \\ community rheumatology ${ }^{1}$
}

${ }^{1}$ Primary Care Musculoskeletal Research Centre, Keele University, Stafford ST5 5BG

${ }^{2}$ School of Health and Rehabilitation, Keele University Correspondence to: N E Foster n.foster@keele.ac.uk

doi:10.1136/bmj.39280.509803.BE

\section{ABSTRACT}

Objective To investigate the benefit of adding acupuncture to a course of advice and exercise delivered by physiotherapists for pain reduction in patients with osteoarthritis of the knee.

Design Multicentre, randomised controlled trial. Setting 37 physiotherapy centres accepting primary care patients referred from general practitioners in the Midlands, United Kingdom.

Participants 352 adults aged 50 or more with a clinical diagnosis of knee osteoarthritis.

Interventions Advice and exercise $(n=116)$, advice and exercise plus true acupuncture ( $n=117)$, and advice and exercise plus non-penetrating acupuncture $(n=119)$. Main outcome measures The primary outcome was change in scores on the Western Ontario and McMaster Universities osteoarthritis index pain subscale at six months. Secondary outcomes included function, pain intensity, and unpleasantness of pain at two weeks, six weeks, six months, and 12 months.

Results Follow-up rate at six months was $94 \%$. The mean (SD) baseline pain score was 9.2 (3.8). At six months mean reductions in pain were 2.28 (3.8) for advice and exercise, 2.32 (3.6) for advice and exercise plus true acupuncture, and 2.53 (4.2) for advice and exercise plus non-penetrating acupuncture. Mean differences in change scores between advice and exercise alone and each acupuncture group were 0.08 (95\% confidence interval -1.0 to 0.9$)$ for advice and exercise plus true acupuncture and 0.25 (-0.8 to 1.3) for advice and exercise plus non-penetrating acupuncture. Similar nonsignificant differences were seen at other follow-up points. Compared with advice and exercise alone there were small, statistically significant improvements in pain intensity and unpleasantness at two and six weeks for true acupuncture and at all follow-up points for nonpenetrating acupuncture.

Conclusion The addition of acupuncture to a course of advice and exercise for osteoarthritis of the knee delivered by physiotherapists provided no additional improvement in pain scores. Small benefits in pain intensity and unpleasantness were observed in both acupuncture groups, making it unlikely that this was due to acupuncture needling effects.
Trial registration Current Controlled Trials ISRCTN88597683.

\section{INTRODUCTION}

Knee pain in older adults is a common disabling problem, managed in the United Kingdom mostly within primary care. ${ }^{1}$ Osteoarthritis is the most likely underlying diagnosis and has been shown by radiography to be present in $70 \%$ of community dwelling adults aged 50 or more with knee pain. ${ }^{2}$ Structural changes before radiography are common in the remainder. $^{3}$ Practice guidelines from the United Kingdom and mainland Europe emphasise the role of education, exercise, and drugs. ${ }^{4-6}$ The recommendations for exercise are underpinned by clinical trials. ${ }^{7-9}$ A recent best evidence summary of systematic reviews concluded that exercise therapy (strengthening, stretching, and functional exercises) compared with no treatment is effective for patients with knee osteoarthritis. ${ }^{10}$ Long term use of oral non-steroidal inflammatory drugs is discouraged ${ }^{11}$ and studies show that patients generally do not like taking drugs. ${ }^{12}$ People with knee osteoarthritis want non-pharmacological options for pain relief $^{13}$ and often choose complementary medicine. ${ }^{14}$

The concept of integrated health care that incorporates conventional and complementary therapies has been promoted. ${ }^{15}$ Between $30 \%$ and $40 \%$ of general practices in England provide access to some form of complementary therapy for their patients, ${ }^{16}$ and acupuncture is one of the most popular options. Each week about $10 \%$ of general practitioners refer patients to acupuncture, or practise it themselves. ${ }^{16}$ More than $10 \%$ of chartered physiotherapists in the United Kingdom $(n=4300)$ are members of an acupuncture group (personal communication, business manager of the Acupuncture Association of Chartered Physiotherapists, January 2007). Despite growing enthusiasm to provide complementary therapy within the UK National Health Service framework and the positive consensus statement from the National Institutes of Health, ${ }^{17}$ the place of acupuncture within mainstream health care remains controversial. Clinical trials of acupuncture have been criticised for small sample sizes, inadequate blinding, and lack of credible sham 
controls and long term follow-up. ${ }^{18-21}$ Recent trials have gone some way to tackling these limitations ${ }^{22-26}$ and systematic reviews conclude that acupuncture is more effective than placebo for osteoarthritis of the knee. ${ }^{2021}$ However, questions about the benefit of adding acupuncture to mainstream, recommended treatments for this population remain unanswered. We have shown that exercise based physiotherapy is more effective than usual primary care for older adults with knee pain, ${ }^{9}$ but no high quality trial has investigated the additional benefit of integrating acupuncture with a recommended treatment such as exercise based physiotherapy for this population. Since physiotherapists, in addition to providing exercise and advice, are one of the largest groups of acupuncture providers within the National Health Service, they are an appropriate group with whom to investigate the effectiveness of integrating acupuncture into mainstream health care.

We carried out a prospective randomised controlled trial within primary care to investigate whether acupuncture is a useful adjunct to exercise based physiotherapy for knee pain in older adults.

\section{METHODS}

Full details of the methods, recruitment, and outcome measures have been published. ${ }^{27}$ Participants were recruited from 37 NHS physiotherapy centres providing services for general practices within the Midlands and Cheshire regions of the United Kingdom between November 2003 and October 2005. Adults aged 50 years or more with knee pain and a clinical diagnosis of knee osteoarthritis were invited to participate. They were referred to physiotherapy by their general practitioner between November 2003 and October 2005 and they had not had acupuncture previously.

We screened referral letters and sent potential participants information leaflets about the study by post. They were subsequently telephoned to make an appointment, further explain the study, confirm eligibility, and obtain consent to participate. The leaflet explained that participants would receive physiotherapy advice and exercise and "may receive acupuncture, using one of two different types of acupuncture needle" without specifying the needles' mode of action (penetrating compared with

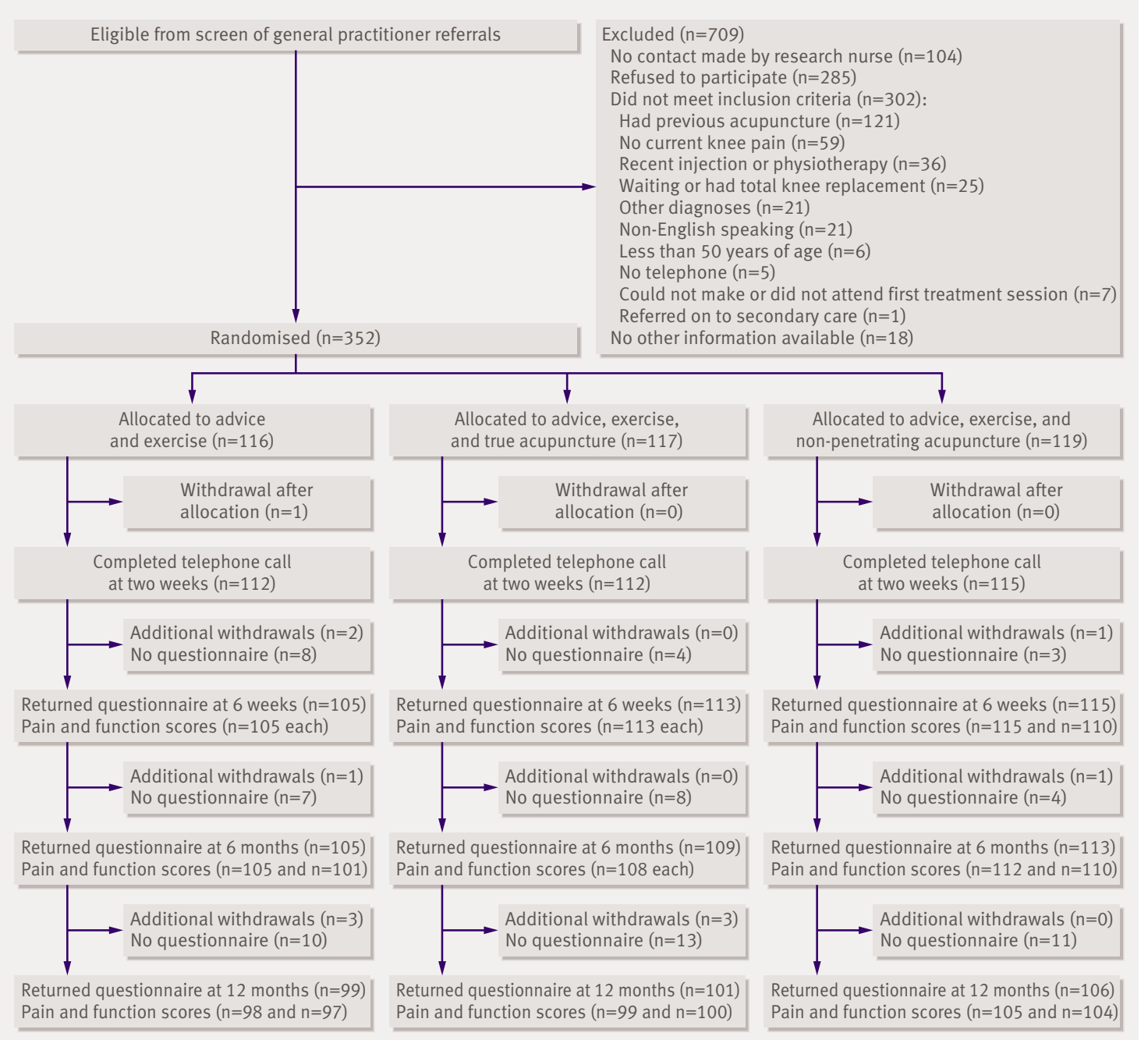


non-penetrating) to maximise the effectiveness of blinding. After consent had been obtained the study nurse carried out a baseline research assessment. Participants then received an initial physiotherapy assessment during which affected knees were examined and conditions other than osteoarthritis (for example, referred pain from the hip or back) were ruled out. Potential acupuncture points were selected and recorded on a form. The first session of advice and exercise was delivered. After this the physiotherapist telephoned an administrator at the research centre to record the selected acupuncture points and to receive a computed generated randomisation group for the patient. This process ensured that the selection of initial acupuncture points, the assessment, and the first advice and exercise session were carried out blind to subsequent treatment allocation.

Using random permuted blocks of 12 (blocked by treatment centre) the participants were randomised to advice and exercise, advice and exercise plus true acupuncture, or advice and exercise plus nonpenetrating acupuncture. Researchers who collected, entered, and analysed data were unaware of treatment allocation. By necessity the physiotherapists delivering the interventions were not blind to allocation.

Table 1 | Baseline characteristics of participants, by treatment group. Values are numbers (percentages) of participants unless stated otherwise

\begin{tabular}{|c|c|c|c|}
\hline Variables & $\begin{array}{l}\text { Advice and exercise } \\
\qquad(n=116)\end{array}$ & $\begin{array}{l}\text { Advice, exercise, and } \\
\text { true acupuncture }(n=117)\end{array}$ & $\begin{array}{l}\text { Advice, exercise, and } \\
\text { non-penetrating acupuncture } \\
\qquad(\mathrm{n}=119)\end{array}$ \\
\hline \multicolumn{4}{|l|}{ Personal details } \\
\hline Mean (SD) age (years) & $63.8(8.3)$ & $63.1(8.7)$ & $62.8(9.4)$ \\
\hline Women & $79(68)$ & $71(61)$ & $66(55)$ \\
\hline \multicolumn{4}{|l|}{ Body mass index: } \\
\hline Underweight or normal («25.0) & $26(22)$ & $25(22)$ & $22(18)$ \\
\hline Overweight (25.0-29.9) & $55(47)$ & $54(47)$ & $49(41)$ \\
\hline Obese (>29.9) & $35(30)$ & $35(31)$ & $48(40)$ \\
\hline \multicolumn{4}{|l|}{ Socioeconomic classification*: } \\
\hline Higher managerial or professional & $9(9)$ & $11(10)$ & $9(9)$ \\
\hline Lower managerial or professional & $19(18)$ & 20 (18) & $18(16)$ \\
\hline Intermediate occupations & $22(21)$ & $15(14)$ & $20(17)$ \\
\hline Self employed & $5(5)$ & $10(9)$ & $8(7)$ \\
\hline Lower supervisory or technical & $3(3)$ & $6(5)$ & $11(10)$ \\
\hline Semiroutine occupations & $23(22)$ & $29(26)$ & $29(25)$ \\
\hline Routine occupations & $24(23)$ & $19(17)$ & $20(17)$ \\
\hline Currently employed & $37(32)$ & $53(45)$ & $48(40)$ \\
\hline \multicolumn{4}{|l|}{ Knee pain and function } \\
\hline Mean (SD) pain score $†$ & $9.1(3.7)$ & $9.3(4.0)$ & $8.9(3.3)$ \\
\hline Mean (SD) function score $†$ & $29.0(12.9)$ & $30.8(13.9)$ & $31.1(12.8)$ \\
\hline \multicolumn{4}{|l|}{ Total duration of knee pain (years): } \\
\hline$\ll 1$ & $47(41)$ & $53(45)$ & $48(40)$ \\
\hline 1 to $<5$ & $38(33)$ & $41(35)$ & $35(29)$ \\
\hline 5 to $<10$ & $14(12)$ & $8(6)$ & $18(15)$ \\
\hline$\geq 10$ & $17(15)$ & $15(13)$ & $18(15)$ \\
\hline \multicolumn{4}{|l|}{ Onset of current problem: } \\
\hline Sudden & $54(47)$ & $51(44)$ & $55(46)$ \\
\hline Gradual & $62(53)$ & $66(56)$ & $64(54)$ \\
\hline Mean (SD) pain severity in past 7 days $\ddagger$ & $5.6(2.2)$ & $6.0(2.3)$ & $6.1(2.1)$ \\
\hline Mean (SD) pain unpleasantness in past 7 days $\ddagger$ & $5.6(2.3)$ & $6.0(2.4)$ & $6.2(2.2)$ \\
\hline Mean (SD) severity of main problem $\ddagger$ & $6.3(2.1)$ & $6.3(2.1)$ & $6.5(2.0)$ \\
\hline \multicolumn{4}{|l|}{ Self efficacy and drugs } \\
\hline Mean (SD) pain§ & $29.0(9.4)$ & $27.2(8.6)$ & $27.3(9.0)$ \\
\hline Mean (SD) other symptoms§ & $36.3(10.5)$ & $34.5(10.8)$ & $33.7(9.9)$ \\
\hline \multicolumn{4}{|l|}{ Used drugs: } \\
\hline Non-steroidal anti-inflammatory drugs & $55(47)$ & $57(49)$ & $63(53)$ \\
\hline Analgesia & $49(42)$ & $49(42)$ & $49(41)$ \\
\hline \multicolumn{4}{|c|}{$\begin{array}{l}\text { Not all numbers add up to totals owing to missing data. } \\
{ }^{\star} \text { Office for National Statistics. }{ }^{39} 40 \\
\text { †Western Ontario and McMaster Universities osteoarthritis index. } \\
\ddagger \text { Numerical rating scale }(0-10) \text {. } \\
\text { §Arthritis self efficacy scale. }\end{array}$} \\
\hline
\end{tabular}




\section{Interventions}

Interventions were delivered within 10 working days of randomisation by 67 physiotherapists trained in acupuncture to at least minimum national standards for membership of the Acupuncture Association of Chartered Physiotherapists. Two thirds of the physiotherapists had been qualified for more than 10 years and over half had been using acupuncture for more than three years. The treatment procedures were agreed and the use of the non-penetrating needle ${ }^{28}$ standardised during training before the trial.

\section{Advice and exercise}

Participants allocated to the advice and exercise group received advice supplemented by a leaflet modelled on the Arthritis Research Campaign leaflet on knee osteoarthritis (www.arc.org.uk). Participants who were receiving non-steroidal anti-inflammatory drugs were permitted to continue with their stable dose. The advice and exercise package was developed from reviews of best evidence, clinical guidelines, a survey of physiotherapy practice for knee pain, ${ }^{29}$ and a consensus workshop. Exercises were individualised using PhysioTools (www.physiotools.net), oriented towards lower limb strengthening, stretching, and balance. This could include concentric, eccentric, and isometric exercise; non-weight bearing exercise; and weight bearing exercise plus a home exercise programme. Intensity was progressed, when appropriate, at each supervised exercise session. The package consisted of up to six sessions of 30 minutes (including the prerandomisation session) over six weeks. Data on participants' self reported adherence to exercise were collected.

\section{Advice and exercise plus true acupuncture}

Participants allocated to advice and exercise plus true acupuncture received acupuncture on traditional Chinese acupuncture points. The acupuncture protocol was based on the concept of adequacy of treatment, ${ }^{21}$ survey results, ${ }^{30}$ a consensus workshop, and recommendations from traditional Chinese protocols. We did not

\begin{tabular}{|c|c|c|c|c|c|}
\hline Patient's response & $\begin{array}{l}\text { Advice and exercise } \\
\qquad(n=112)\end{array}$ & $\begin{array}{l}\text { Advice, exercise, and } \\
\text { true acupuncture } \\
(n=112)\end{array}$ & $\begin{array}{l}P \text { value } \\
\left(x^{2} \text { test for }\right. \\
\text { trend }) \dagger\end{array}$ & $\begin{array}{l}\text { Advice, exercise, and } \\
\text { non-penetrating } \\
\text { acupuncture }(n=115)\end{array}$ & $\begin{array}{l}P \text { value } \\
\left(X^{2} \text { test for }\right. \\
\text { trend }) \ddagger\end{array}$ \\
\hline \multicolumn{6}{|c|}{$\begin{array}{l}\text { Confident that treatment can help } \\
\text { problem?: }\end{array}$} \\
\hline Very & $26(23)$ & $27(24)$ & \multirow{5}{*}{0.01} & $38(33)$ & \multirow{5}{*}{0.0001} \\
\hline Quite & $44(39)$ & $64(57)$ & & $60(53)$ & \\
\hline Neither & $18(16)$ & $12(11)$ & & $10(9)$ & \\
\hline Not very & $21(19)$ & $7(6)$ & & $5(4)$ & \\
\hline Not at all & $3(3)$ & $2(2)$ & & $1(1)$ & \\
\hline Missing data & 0 & 0 & & 1 & \\
\hline \multicolumn{6}{|c|}{$\begin{array}{l}\text { Recommend treatment to friend with } \\
\text { similar knee problem?: }\end{array}$} \\
\hline Very confident & $29(26)$ & $34(30)$ & \multirow{5}{*}{0.2} & $41(36)$ & \multirow{5}{*}{0.03} \\
\hline Quite confident & $61(54)$ & $58(52)$ & & $62(54)$ & \\
\hline Neither & $8(7)$ & $15(13)$ & & $4(4)$ & \\
\hline Not very confident & $12(11)$ & $5(4)$ & & $5(4)$ & \\
\hline Not at all confident & $2(2)$ & $0(0)$ & & $2(2)$ & \\
\hline Missing data & 0 & 0 & & 1 & \\
\hline \multicolumn{6}{|c|}{ Does treatment make sense to you?: } \\
\hline Very logical & $46(41)$ & $32(29)$ & \multirow{5}{*}{0.006} & $45(39)$ & \multirow{5}{*}{0.4} \\
\hline Quite logical & $53(47)$ & $54(48)$ & & $51(45)$ & \\
\hline No opinion & $5(4)$ & $6(5)$ & & $4(4)$ & \\
\hline Not very logical & $7(6)$ & $18(16)$ & & $13(11)$ & \\
\hline Not at all logical & $1(1)$ & $2(2)$ & & $1(1)$ & \\
\hline Missing data & 0 & 0 & & 1 & \\
\hline \multicolumn{6}{|c|}{$\begin{array}{l}\text { Do you think treatment would be } \\
\text { successful in treating other problems? }\end{array}$} \\
\hline Very & $17(15)$ & $17(15)$ & \multirow{5}{*}{0.3} & $29(25)$ & \multirow{5}{*}{0.03} \\
\hline Quite & $61(55)$ & $68(61)$ & & $56(49)$ & \\
\hline No opinion & $27(24)$ & $25(22)$ & & $29(25)$ & \\
\hline Not very & $5(5)$ & $1(1)$ & & $0(0)$ & \\
\hline Not at all & $2(2)$ & $1(1)$ & & $0(0)$ & \\
\hline Missing data & 0 & 0 & & 1 & \\
\hline
\end{tabular}

*Adapted from Vincent and Lewith. ${ }^{36}$

†Advice and exercise compared with advice, exercise, and true acupuncture.

$\ddagger$ Advice and exercise compared with advice, exercise, and non-penetrating acupuncture. 
allow moxibustion, cupping, herbs, or electroacupuncture. For each individualised treatment session between six and 10 acupuncture points from 16 commonly used local and distal points were selected. Local points were Sp 9, Sp 10, St 34, St 35, St 36, Xiyan, Gb 34, and trigger points. Distal points were LI 4, TH 5, Sp 6, Liv 3, St 44, $\mathrm{Ki} 3$, BI 60, and Gb 41. Sterilised disposable steel needles $(30 \times 0.3 \mathrm{~mm})$ were used; the depth of insertion was between $5 \mathrm{~mm}$ and $25 \mathrm{~mm}$, depending on the points selected. Needles were manipulated to achieve the de qi sensation (for example, aching, warm or tingling sensation) and the therapists recorded the sensations that patients reported. The protocol permitted 25 to 35 minutes between insertion of the last needle and stopping treatment. The therapists revisited and manipulated the needles as appropriate. If the de qi sensation was no longer present the therapists were expected to use stronger manipulation, either rotation or thrust and withdraw techniques, to elicit it. In addition to the prerandomisation advice and exercise session the package consisted of up to six treatment sessions over three weeks, during which both the true acupuncture and the advice and exercise treatments were delivered.

\section{Advice and exercise plus non-penetrating acupuncture}

Participants randomised to receive advice and exercise plus non-penetrating acupuncture ${ }^{28}$ had acupuncture delivered through needles with a blunt tip. The shaft of these needles collapses into the handle, creating an illusion of insertion. They meet the recommendations for acceptable controls for acupuncture research ${ }^{18}$ and have been used in previous trials. ${ }^{2231}$ Some authors believe that a small acupressure effect may be induced by this method. ${ }^{31}$ The same protocol was used as for true acupuncture, thus all criteria for harnessing nonspecific effects were included (same contact time and interaction between therapist and patient, manual contact during the search for acupuncture points, and attention to elicited sensations). No attempt was made to elicit the de qi sensation but participants were told they may experience sensations and to report what they felt. In addition to the prerandomisation advice and exercise session the package consisted of up to six treatment sessions over three weeks, during which both the non-penetrating acupuncture and the advice and exercise treatments were delivered.

\section{Outcomes}

Full details of the outcome measures are published elsewhere. ${ }^{27}$ Follow-up was at two weeks (by telephone), six weeks, and six and 12 months (by postal questionnaire) after randomisation. The primary outcome measure was change at six months in the pain subscale score of the Western Ontario and McMaster Universities osteoarthritis index (Likert 3.0). ${ }^{32}$ This subscale has been shown to be reliable for use within postal surveys. ${ }^{33}$ Secondary outcomes at six months included the function subscale score of the Western Ontario and McMaster Universities osteoarthritis index, participants' global assessment of change compared with baseline, pain severity and unpleasantness, severity of patient nominated main functional problem, arthritis self efficacy, satisfaction with care, and adherence with exercises, and all measures at six weeks and 12 months. Side effects of treatment, adverse events, and use of cointerventions were also recorded. We identified the proportion of each group who achieved a clinically significant response according to criteria from the outcome measures in Rheumatology and Osteoarthritis Research Society international initiative (OMERACT-OARSI). ${ }^{3435}$

Physiotherapists recorded the number and duration of treatment sessions, the exercises prescribed, the location and number of acupuncture points (when applicable), and any adverse reactions. In the acupuncture groups, therapists recorded the sensations that participants reported. This information was subsequently categorised by four acupuncture experts (three independent of the study team), blinded to treatment allocation, to identify those most likely to represent the de qi sensation. Acceptability and credibility of the intervention groups were evaluated during a telephone call two weeks after the start of treatment and at six weeks by questionnaire, using items adapted from previous literature. ${ }^{36}$

\section{Statistical analysis}

We assumed a $45 \%$ improvement in pain measured by the pain subscale at six months in participants

\begin{tabular}{|c|c|c|c|c|c|}
\hline Variable & $\begin{array}{l}\text { Advice and } \\
\text { exercise } \\
(n=112)\end{array}$ & $\begin{array}{l}\text { Advice, exercise, } \\
\text { and true acupuncture } \\
(n=112)\end{array}$ & $\begin{array}{c}P \text { value* } \\
\left(X^{2} \text { test for trend) }\right.\end{array}$ & $\begin{array}{l}\text { Advice, exercise, } \\
\text { and non-penetrating } \\
\text { acupuncture }(n=115)\end{array}$ & $\begin{array}{c}\text { P value } \dagger \\
\text { ( } \mathrm{X}^{2} \text { test for trend) }\end{array}$ \\
\hline \multicolumn{6}{|c|}{$\begin{array}{l}\text { I have been doing my exercises as } \\
\text { often as advised: }\end{array}$} \\
\hline Strongly agree & $35(32)$ & $41(37)$ & \multirow{5}{*}{0.2} & 44 (39) & \multirow{5}{*}{0.7} \\
\hline Agree & $34(31)$ & 37 (33) & & $27(24)$ & \\
\hline Not sure & $0(0)$ & $2(2)$ & & $0(0)$ & \\
\hline Disagree & $40(36)$ & $31(28)$ & & $41(36)$ & \\
\hline Strongly disagree & $2(2)$ & $1(1)$ & & $2(2)$ & \\
\hline Missing data & 1 & 0 & - & 1 & - \\
\hline
\end{tabular}

*Advice and exercise compared with advice, exercise, and true acupuncture.

†Advice and exercise compared with advice, exercise, and non-penetrating acupuncture. 
randomised to the advice and exercise group. The sample size calculation was based on the ability to detect a $65 \%$ improvement in the two groups also receiving acupuncture (an additional improvement of 20\%). ${ }^{37}$ A minimum of 270 participants with data at six months would be sufficient to detect these effects with $80 \%$ power at a $5 \%$ significance level (two tailed). ${ }^{38}$ To allow for dropouts we inflated this value by $30 \%$ and hence aimed to randomise 350 participants.

Analysis was by intention to treat. Estimates of the treatment effects for numerical data are presented as difference in mean change, with 95\% confidence intervals (advice and exercise plus acupuncture groups minus advice and exercise alone group) and $\chi^{2}$ tests (heterogeneity test for binary data or linear trend test for ordinal data). We carried out exploratory sensitivity analyses of the mean pain subscale scores by adjusting for baseline characteristics using multiple linear regression. A priori subgroup analyses included comparing those participants in the advice and exercise plus true acupuncture group who had 50\% or more treatment sessions in which the de qi sensation was elicited with those with less than $50 \%$, and comparing the effect of treatment in those who had severe knee pain or disability at baseline. External validity was assessed by comparing demographic characteristics of patients not randomised with those of participants and the participants' recruitment characteristics and treatment allocation across high and low recruiting centres.

ET carried out statistical analyses using Stata 7.0. The trial was monitored by an independent steering and data monitoring committee and no interim analyses were undertaken.

\begin{tabular}{|c|c|c|c|c|c|c|c|}
\hline & $\begin{array}{l}\text { Advice and } \\
\text { exercise }\end{array}$ & $\begin{array}{c}\text { Advice, } \\
\text { exercise, and } \\
\text { true } \\
\text { acupuncture }\end{array}$ & $\begin{array}{l}\text { Mean difference } † \\
(95 \% \mathrm{Cl})\end{array}$ & Pvalue $\neq$ & $\begin{array}{l}\text { Advice, exercise, } \\
\text { and non- } \\
\text { penetrating } \\
\text { acupuncture }\end{array}$ & $\begin{array}{l}\text { Mean difference } \dagger \\
(95 \% \mathrm{Cl})\end{array}$ & $P$ value $\ddagger$ \\
\hline \multicolumn{8}{|l|}{ Pain } \\
\hline \multicolumn{8}{|l|}{ Six weeks: } \\
\hline No analysed & 105 & 113 & & & 115 & & \\
\hline Absolute score & $6.86(4.2)$ & $6.38(4.1)$ & & & $5.98(4.3)$ & & \\
\hline Crude change score§ & $2.10(3.5)$ & $2.83(4.0)$ & $0.74(-0.3$ to 1.7$)$ & 0.1 & $3.02(3.6)$ & $0.93(0.0$ to 1.9$)$ & 0.05 \\
\hline Adjusted change scoref & & & $0.69(-0.2$ to 1.6$)$ & 0.1 & & $0.88(0.0$ to 1.8$)$ & 0.06 \\
\hline \multicolumn{8}{|l|}{ Six months: } \\
\hline No analysed & 105 & 108 & & & 112 & & \\
\hline Absolute score & $6.78(4.5)$ & $7.07(4.4)$ & & & $6.50(4.8)$ & & \\
\hline Crude change score§ & $2.28(3.8)$ & $2.32(3.6)$ & $0.04(-1.0$ to 1.0$)$ & 0.9 & $2.53(4.2)$ & $0.25(-0.8$ to 1.3$)$ & 0.6 \\
\hline Adjusted change scoref & & & $-0.08(-1.0$ to 0.9$)$ & 0.9 & & $0.25(-0.8$ to 1.3$)$ & 0.6 \\
\hline \multicolumn{8}{|l|}{12 months: } \\
\hline No analysed & 98 & 99 & & & 105 & & \\
\hline Absolute score & $6.29(4.7)$ & $6.84(4.7)$ & & & $6.16(4.8)$ & & \\
\hline Crude change score§ & $2.57(4.3)$ & $2.37(4.2)$ & $-0.20(-1.4$ to 1.0$)$ & 0.7 & $2.82(4.1)$ & $0.25(-0.9$ to 1.4$)$ & 0.7 \\
\hline Adjusted change scoref & & & $-0.42(-1.5$ to 0.7$)$ & 0.5 & & $0.23(-0.9$ to 1.3$)$ & 0.7 \\
\hline \multicolumn{8}{|l|}{ Function } \\
\hline \multicolumn{8}{|l|}{ Six weeks: } \\
\hline No analysed & 105 & 113 & & & 110 & & \\
\hline Absolute score & $22.34(14.9)$ & $22.38(14.5)$ & & & $22.14(15.7)$ & & \\
\hline Crude change score§ & $6.21(11.4)$ & $8.18(11.5)$ & $1.97(-1.1$ to 5.0$)$ & 0.2 & $9.32(11.4)$ & $3.11(0.0$ to 6.2$)$ & 0.05 \\
\hline Adjusted change scoref & & & $1.46(-1.5$ to 4.4$)$ & 0.3 & & $2.49(-0.6$ to 5.5$)$ & 0.1 \\
\hline \multicolumn{8}{|l|}{ Six months: } \\
\hline No analysed & 101 & 108 & & & 110 & & \\
\hline Absolute score & $24.36(15.6)$ & $24.93(16.0)$ & & & $23.83(16.9)$ & & \\
\hline Crude change score§ & $4.60(11.4)$ & $6.25(12.1)$ & $1.66(-1.5$ to 4.9$)$ & 0.3 & $7.13(13.1)$ & $2.54(-0.8$ to 5.9$)$ & 0.1 \\
\hline Adjusted change scoreף & & & $0.92(-2.2$ to 4.0$)$ & 0.6 & & $2.39(-0.9$ to 5.6$)$ & 0.2 \\
\hline \multicolumn{8}{|l|}{12 months: } \\
\hline No analysed & 97 & 100 & & & 104 & & \\
\hline Absolute score & $23.16(15.8)$ & $23.83(16.5)$ & & & $22.47(16.7)$ & & \\
\hline Crude change score§ & $5.36(11.9)$ & $6.61(13.8)$ & $1.24(-2.3$ to 4.5$)$ & 0.5 & $8.24(13.5)$ & $2.87(-0.7$ to 6.4$)$ & 0.1 \\
\hline Adjusted change scoref & & & $0.23(-3.2$ to 3.6$)$ & 0.9 & & $2.52(-0.9$ to 6.0$)$ & 0.2 \\
\hline $\begin{array}{l}\text { *Scores on Western Ontario } \\
\text { †Difference in mean scores } \\
\text { †Derived from linear regress } \\
\text { \$Change in score from basel } \\
\text { \Mean difference adjusted } \mathrm{f}\end{array}$ & $\begin{array}{l}\text { d McMaster Ur } \\
\text { vice and exerc } \\
\text { ecruitment ag }\end{array}$ & $\begin{array}{l}\text { iversities osteo } \\
\text { se plus acupun } \\
\text {, sex, duration }\end{array}$ & $\begin{array}{l}\text { arthritis index. } \\
\text { cture group minus ac } \\
\text { of pain, and scores } f\end{array}$ & pain or $\mathrm{fu}$ & $\begin{array}{l}\text { ise group). } \\
\text { tion. }\end{array}$ & & \\
\hline
\end{tabular}




\section{RESULTS}

Overall, 1061 potentially eligible participants were identified, of whom $709(66.8 \%)$ were ineligible or did not want to participate (figure). In total 352 $(33.2 \%)$ patients were randomised: 116 to advice and exercise, 117 to advice and exercise plus true acupuncture, and 119 to advice and exercise plus non-penetrating acupuncture. Thirty seven physiotherapy centres recruited at least one patient (maximum 29 per site). Baseline characteristics of participants (mean age 63 years, 61\% women) were similar between the groups (table 1).

Those patients who were screened but not randomised $(\mathrm{n}=709)$ were slightly older than those randomised (65 v 63 years), but both groups had $61 \%$ of women. Treatment allocation and recruitment characteristics were similar between the higher $(\geq 18$ patients; $n=177$ patients) and the lower ( $\leq 17$ patients; $\mathrm{n}=175$ patients) recruiting centres (data not shown).

Four participants in the advice and exercise group and two in the advice and exercise plus non-penetrating acupuncture group withdrew permission for further contact in the period up to the six months' follow-up. In the 346 eligible for contact at six months the response rate was $94 \%$ for advice and exercise, $93 \%$ for advice and exercise plus true acupuncture, and $97 \%$ for advice and exercise plus non-penetrating acupuncture. Nineteen participants did not return the questionnaire at six months. Non-responders were more likely to be men (47\% v 39\%), to be younger (mean age $60 v 63$ years), and to have slightly lower baseline scores for pain and function (pain: $8.7 v 9.2$, function: $27.8 v 30.5$ ).

Treatments were in line with the study protocols. A few participants violated the protocol: three in the advice and exercise group and two in the advice and exercise plus non-penetrating acupuncture group. No adverse events occurred in the advice and exercise group or in the advice and exercise plus nonpenetrating acupuncture group. Five adverse events were reported for participants receiving true acupuncture (pain, sleepiness, fainting, nausea, and swelling around the treated knee).

\begin{tabular}{|c|c|c|c|c|c|}
\hline Variables & $\begin{array}{l}\text { Advice and } \\
\text { exercise }\end{array}$ & $\begin{array}{l}\text { Advice, exercise, and } \\
\text { true acupuncture }\end{array}$ & $\begin{array}{c}\text { P value* } \\
\left(\mathrm{X}^{2} \text { test for trend }\right)\end{array}$ & $\begin{array}{l}\text { Advice, exercise, and } \\
\text { non-penetrating } \\
\text { acupuncture }\end{array}$ & $\begin{array}{c}\text { P value } \dagger \\
\left(x^{2} \text { test for trend }\right)\end{array}$ \\
\hline \multicolumn{6}{|l|}{ Two weeks: } \\
\hline Much better & $14(13)$ & $25(22)$ & \multirow{5}{*}{0.02} & $29(25)$ & \multirow{5}{*}{0.008} \\
\hline Better & $55(49)$ & $58(52)$ & & $53(46)$ & \\
\hline No change & $34(30)$ & $25(22)$ & & $28(25)$ & \\
\hline Worse & $7(6)$ & $2(2)$ & & $4(4)$ & \\
\hline Much worse & $2(2)$ & $2(2)$ & & $0(0)$ & \\
\hline Missing data & 0 & 0 & & 1 & \\
\hline \multicolumn{6}{|l|}{ Six weeks: } \\
\hline Complete recovery & $4(4)$ & $1(1)$ & & $4(4)$ & \\
\hline Much better & $25(24)$ & $33(30)$ & \multirow{5}{*}{0.3} & $42(37)$ & \multirow{5}{*}{0.2} \\
\hline Better & $42(40)$ & $52(46)$ & & $36(32)$ & \\
\hline No change & $24(23)$ & $23(21)$ & & $25(22)$ & \\
\hline Worse & $8(8)$ & $2(2)$ & & $5(4)$ & \\
\hline Much worse & $1(1)$ & $1(1)$ & & $2(2)$ & \\
\hline Missing data & 1 & 1 & & 1 & \\
\hline \multicolumn{6}{|l|}{ Six months: } \\
\hline Complete recovery & $4(4)$ & $3(3)$ & & $6(5)$ & \\
\hline Much better & $24(23)$ & $23(21)$ & \multirow{5}{*}{0.9} & $38(34)$ & \multirow{5}{*}{0.2} \\
\hline Better & $28(27)$ & $33(31)$ & & $23(21)$ & \\
\hline No change & $33(31)$ & $33(31)$ & & $29(26)$ & \\
\hline Worse & $12(11)$ & $12(11)$ & & $13(12)$ & \\
\hline Much worse & $4(4)$ & $4(4)$ & & $3(3)$ & \\
\hline Missing data & 0 & 1 & & 1 & \\
\hline \multicolumn{6}{|l|}{12 months: } \\
\hline Complete recovery & $11(11)$ & $4(4)$ & & $5(5)$ & \\
\hline Much better & 19 (19) & $20(20)$ & \multirow{6}{*}{0.7} & $31(29)$ & \multirow{6}{*}{0.8} \\
\hline Better & $21(21)$ & 39 (39) & & $26(25)$ & \\
\hline No change & $30(30)$ & $15(15)$ & & $25(24)$ & \\
\hline Worse & $12(12)$ & $17(17)$ & & $14(13)$ & \\
\hline Much worse & $6(6)$ & $6(6)$ & & $5(5)$ & \\
\hline Missing data & 0 & 0 & & 0 & \\
\hline
\end{tabular}

*Advice and exercise compared with advice, exercise, and true acupuncture.

†Advice and exercise compared with advice, exercise, and non-penetrating acupuncture. 
Table 6 | Response to criteria from the outcome measures in Rheumatology and Osteoarthritis Research Society international initiative at follow-up. Values are numbers (percentages) of participants unless stated otherwise

\begin{tabular}{|c|c|c|c|c|c|}
\hline Variables & Advice and exercise & $\begin{array}{c}\text { Advice, exercise, } \\
\text { and true } \\
\text { acupuncture }\end{array}$ & $\begin{array}{c}\text { P value* } \\
\left(X^{2} \text { test for trend }\right)\end{array}$ & $\begin{array}{l}\text { Advice, exercise, } \\
\text { and non-penetrating } \\
\text { acupuncture }\end{array}$ & $\begin{array}{c}\text { P value } \dagger \\
\left(X^{2} \text { test for trend }\right)\end{array}$ \\
\hline \multicolumn{6}{|l|}{ Six weeks: } \\
\hline High & 30 & 39 & \multirow{2}{*}{0.1} & 51 & \multirow{2}{*}{0.1} \\
\hline Improvement & 24 & 31 & & 20 & \\
\hline Total response & $54(51)$ & $70(62)$ & & $71(62)$ & \\
\hline \multicolumn{6}{|l|}{ Six months: } \\
\hline High & 31 & 29 & \multirow{2}{*}{0.4} & 38 & \multirow{2}{*}{0.2} \\
\hline Improvement & 14 & 26 & & 21 & \\
\hline Total response & $45(43)$ & $55(50)$ & & $59(52)$ & \\
\hline \multicolumn{6}{|l|}{12 months: } \\
\hline High & 37 & 31 & \multirow{2}{*}{0.6} & 41 & \multirow{2}{*}{0.3} \\
\hline Improvement & 11 & 22 & & 18 & \\
\hline Total response & $48(48)$ & $53(52)$ & & $59(56)$ & \\
\hline
\end{tabular}

The median (range) number of treatment sessions was in line with the study protocols: $6(1-9)$ in the advice and exercise group, 7 (4-9) in the advice and exercise plus true acupuncture group, and 7 (1-8) in the advice and exercise plus non-penetrating acupuncture group. In both acupuncture groups the acupuncture treatment was used in a median of six sessions. The de qi sensation was reported at least once for $95(83 \%)$ participants receiving true acupuncture and $65(55 \%)$ receiving non-penetrating acupuncture. Of these, $67(71 \%)$ and $29(45 \%)$ reported de qi during at least half of their treatment sessions.

\section{Intervention credibility and exercise compliance}

Table 2 summarises treatment credibility two weeks after treatment started. Participants receiving either acupuncture intervention were significantly more confident that treatment could help their knee problem than those receiving advice and exercise alone.

Most participants had treatment sessions that included supervised exercises $(85 \%$ for advice and exercise, $77 \%$ for advice and exercise plus true acupuncture, and $78 \%$ for advice and exercise plus non-penetrating acupuncture) and a home exercise programme $(89 \%, 91 \%$, and $95 \%)$. Self reported compliance with exercise at two weeks was $63 \%, 70 \%$, and $64 \%$ (table 3). Compliance remained above $50 \%$ in each group over the 12 months' follow-up.

\section{Outcome measures}

At six months no statistically significant differences were found in change on the pain subscale from baseline between the groups receiving acupuncture in addition to advice and exercise compared with the group receiving advice and exercise alone (table 4).

At six weeks the advice and exercise plus nonpenetrating acupuncture group reported small but significantly greater improvements in pain than did the advice and exercise group (mean difference 0.88 ,
$95 \%$ confidence interval 0.0 to 1.8$)$. At 12 months no statistically significant differences were found between the groups (table 4). At two weeks statistically significant trends were found in favour of better global outcome for each of the acupuncture groups compared with the advice and exercise alone group (table 5).

No other statistically significant differences were found in the changes in function scores, global assessment, or response status according to the OMERACTOARSI criteria between the three groups at any followup points, or in the adjusted analyses (tables 5 and 6).

Statistically significant differences were found between the groups in pain intensity and unpleasantness (table 7). The results at two and six weeks for pain intensity and at six weeks for change in pain unpleasantness favoured both groups receiving acupuncture. The results at six and 12 months for both pain intensity and pain unpleasantness favoured the group receiving advice and exercise plus nonpenetrating acupuncture. Satisfaction with care was significantly greater for participants receiving advice and exercise plus non-penetrating acupuncture than for those receiving advice and exercise alone (table 8). No statistically significant differences were seen between advice and exercise plus true acupuncture and advice and exercise plus nonpenetrating acupuncture for any other of the outcomes measured (data not shown).

Exploratory subgroup analyses showed no significant differences in change scores for pain or function between participants in the advice and exercise plus true acupuncture group reporting de qi during more than $50 \%$ of treatment sessions compared with those who reported de qi less often. Those with severe pain or disability at baseline showed similar change scores in each of the treatment groups (data not shown). No significant differences were found between groups in the number of reported general practitioner consultations over six months (advice and exercise 
$25 \%$, advice and exercise plus true acupuncture $19 \%$, and advice and exercise plus non-penetrating acupuncture $20 \%$ ) or in the use of non-steroidal anti-inflammatory or simple analgesics.

\section{DISCUSSION}

Acupuncture delivered by physiotherapists as part of an integrated package of health care with advice and exercise, for older adults with osteoarthritis of the knee, provided no additional improvement in pain scores compared with advice and exercise alone measured on the Western Ontario and McMaster Universities osteoarthritis index at six and 12 months. Small benefits were shown for pain intensity and unpleasantness but these effects were greater and sustained for longer in the group receiving non-penetrating acupuncture than in the group receiving true acupuncture. This finding makes it unlikely that the observed effects were due to needling effects of needle penetration, manual stimulation throughout treatment, and elicitation of the de qi sensation.

\section{Comparison with other studies}

Our trial is important for two reasons. Firstly, it provides new information on the effectiveness of acupuncture for knee osteoarthritis in older adults, delivered in addition to an evidence based package of physiotherapy care within mainstream health care. Previous trials of acupuncture for knee osteoarthritis have compared true acupuncture with sham acupuncture (including off-point needling), ${ }^{22}$ ongoing stable medication, ${ }^{41}$ waiting list controls, ${ }^{24}$ or education alone. ${ }^{23}$ We decided not to include a no treatment group as this would be difficult to justify given that guidelines and previous trials recommend advice and exercise for older adults with knee osteoarthritis. Our procedures differed in several important ways from those in previous trials. We used fewer treatment sessions: six acupuncture treatments compared with 10,12, 15, and 24 in previous studies. ${ }^{23-26}$ Although it can be argued that this may have rendered the true acupuncture intervention suboptimal, we found no differences between true and non-penetrating acupuncture after

Table 7 | Change in knee pain and function and psychological measures at follow-up. Values are means (standard deviations) unless stated otherwise

\begin{tabular}{|c|c|c|c|c|c|c|c|}
\hline End point & $\begin{array}{l}\text { Advice and } \\
\text { exercise }\end{array}$ & $\begin{array}{l}\text { Advice, } \\
\text { exercise, } \\
\text { and true } \\
\text { acupunc- } \\
\text { ture }\end{array}$ & $\begin{array}{l}\text { Difference } \\
(95 \% \mathrm{Cl})^{\star}\end{array}$ & $P$ value & $\begin{array}{c}\text { Advice, } \\
\text { exercise, and } \\
\text { non-penetrating } \\
\text { acupuncture }\end{array}$ & $\begin{array}{l}\text { Difference } \\
(95 \% \mathrm{Cl})^{\star}\end{array}$ & $P$ value \\
\hline \multicolumn{8}{|c|}{ Knee pain and function } \\
\hline \multicolumn{8}{|c|}{ Change in pain intensity†: } \\
\hline 2 weeks & $0.27(2.2)$ & $1.31(2.2)$ & $1.12(0.5$ to 1.7$)$ & $<0.0001$ & $1.51(2.1)$ & $1.24(0.7$ to 1.8$)$ & $<0.0001$ \\
\hline 6 weeks & $0.90(2.5)$ & $1.81(2.4)$ & 0.98 (0.3 to 1.6$)$ & 0.004 & $2.18(2.5)$ & 1.27 (0.6 to 1.9$)$ & $<0.0001$ \\
\hline 6 months & $0.95(2.6)$ & $1.28(2.4)$ & $0.32(-0.4$ to 1.0$)$ & 0.4 & $1.95(2.6)$ & 1.01 (0.3 to 1.7$)$ & 0.006 \\
\hline 12 months & $1.21(2.9)$ & $1.60(2.6)$ & $0.34(-0.4$ to 1.1$)$ & 0.4 & $2.02(2.6)$ & 0.84 (0.1 to 1.6$)$ & 0.03 \\
\hline \multicolumn{8}{|c|}{$\begin{array}{l}\text { Change in pain } \\
\text { unpleasantnesst: }\end{array}$} \\
\hline 6 weeks & $1.08(2.5)$ & $1.91(2.4)$ & $0.90(0.2$ to 1.6$)$ & 0.007 & $2.23(2.5)$ & 1.09 (0.4 to 1.8$)$ & 0.002 \\
\hline 6 months & $0.94(2.6)$ & $1.22(2.5)$ & $0.30(-0.4$ to 1.0$)$ & 0.4 & $2.07(2.8)$ & 1.12 (0.4 to 1.9$)$ & 0.003 \\
\hline 12 months & $1.20(3.0)$ & $1.66(2.8)$ & $0.43(-0.4$ to 1.2$)$ & 0.3 & $2.15(2.8)$ & 0.94 (0.2 to 1.7$)$ & 0.02 \\
\hline \multicolumn{8}{|c|}{$\begin{array}{l}\text { Change in severity of main } \\
\text { problem†: }\end{array}$} \\
\hline 6 weeks & $1.39(2.6)$ & $1.88(2.4)$ & $0.58(-0.1$ to 1.3$)$ & 0.09 & $2.13(2.4)$ & $0.72(0.1$ to 1.4$)$ & 0.04 \\
\hline 6 months & $1.13(2.7)$ & $1.23(2.5)$ & $0.12(-0.6$ to 0.8$)$ & 0.7 & $1.76(2.8)$ & $0.66(-0.1$ to 1.4$)$ & 0.08 \\
\hline 12 months & $1.36(2.6)$ & $1.60(2.7)$ & $0.15(-0.6$ to 0.9$)$ & 0.7 & $1.89(2.7)$ & $0.52(-0.2$ to 1.3$)$ & 0.2 \\
\hline
\end{tabular}

Psychological measures

Change in pain on arthritis self

efficacy scale†:

\begin{tabular}{|c|c|c|c|c|c|c|c|}
\hline 6 weeks & $0.71(9.5)$ & $-1.46(9.3)$ & $-2.19(-4.8$ to 0.4$)$ & 0.1 & $-2.54(10.4)$ & $-2.99(-5.7$ to -0.3$)$ & 0.03 \\
\hline 6 months & $1.64(11.1)$ & $-0.62(9.8)$ & $-2.36(-5.3$ to 0.5$)$ & 0.1 & $-1.43(11.3)$ & $-2.77(-5.9$ to 0.3$)$ & 0.08 \\
\hline 12 months & $0.91(12.1)$ & $0.33(12.0)$ & $-0.64(-4.2$ to 2.9$)$ & 0.7 & $0.08(10.3)$ & $-0.71(-3.9$ to 2.5$)$ & 0.7 \\
\hline \multicolumn{8}{|c|}{$\begin{array}{l}\text { Change in other symptoms on } \\
\text { arthritis self efficacy scalet: }\end{array}$} \\
\hline 6 weeks & $-0.32(10.0)$ & $-1.83(10.1)$ & $-1.42(-4.2$ to 1.3$)$ & 0.3 & $-3.38(10.4)$ & $\begin{array}{c}-2.74(-5.5 \text { to } \\
0.02)\end{array}$ & 0.05 \\
\hline 6 months & $0.38(11.2)$ & $-0.68(11.2)$ & $-1.01(-4.1$ to 2.1$)$ & 0.5 & $-1.88(12.0)$ & $-2.09(-5.3$ to 1.1$)$ & 0.2 \\
\hline 12 months & $0.55(11.5)$ & $-0.12(13.9)$ & $-0.47(-4.2$ to 3.3$)$ & 0.8 & $-1.93(11.5)$ & $-2.48(-5.8$ to 0.8$)$ & 0.1 \\
\hline
\end{tabular}

*Mean difference (advice and exercise plus acupuncture group minus advice and exercise group) adjusted for recruitment age, sex, and duration of pain.

†Change in score from baseline. 
six treatments. We included participants with a clinical diagnosis of knee osteoarthritis, who are the patients seen in primary care, rather than those with a confirmed radiological diagnosis only, as used in other trials. ${ }^{24-26}$ We also used the credible acupuncture control of non-penetrating acupuncture at the same points as the true acupuncture rather than minimal depth needling at predefined distant non-acupuncture points. There is much debate within the acupuncture literature about the validity of sham acupuncture, and given that a considerable proportion of participants in our non-penetrating acupuncture group reported sensations fitting the normal descriptions of de qi we cannot consider this intervention as inert. Only one high quality trial included physiotherapy in an integrated package with acupuncture. ${ }^{25}$ In that trial all treatment groups (true acupuncture, minimal needling acupuncture, and conservative therapy consisting of visits to a general practitioner and drugs) could have up to six sessions with a physiotherapist although these were actually received by only a minority of participants. Although procedural differences make direct comparisons between trials difficult, the treatment response in our control group (a course of advice and exercise) was considerably higher $(51 \%$ at six weeks, $43 \%$ at six months, and $48 \%$ at 12 months) than in the control groups of previous trials $(29 \%, 6.5 \%$, and $30 \%$ at six months or equivalent). ${ }^{232526}$ Thus it is clear that the course of six sessions of advice plus individualised and supervised exercise in the current trial was considerably more effective than the control interventions used in previous acupuncture studies. We have shown that acupuncture offers limited additional benefits when delivered alongside an effective package of physiotherapy led advice and exercise.

Secondly, this trial adds to the debate about acupuncture's mechanisms of action. Participants were not told they may receive a sham intervention (rather they were told they may receive one of two forms of acupuncture), the credibility of the interventions was high, and participants were not asked to guess the treatment they had received. We have shown that there are no differences when a credible, nonpenetrating acupuncture treatment, delivered under strict participant blinded conditions, and true acupuncture, involving needle penetration, manual stimulation, and elicitation of the de qi sensation are added to a course of advice and exercise. Indeed patient satisfaction, credibility of intervention at six weeks, and reduction in pain intensity and unpleasantness were significantly greater for the advice and exercise plus non-penetrating acupuncture group than for the advice and exercise group but not for the advice and exercise plus true acupuncture group. We cannot explain these findings. One possibility is that non-penetrating acupuncture harnesses some of the benefits of acupuncture, such as acupressure or participants' expectations of benefit, without any adverse or unpleasant side effects, such as minor bleeding, bruising, or pain. Our findings make it difficult to sustain the argument that the observed effects of acupuncture are explained by specific physiological

\begin{tabular}{|c|c|c|c|c|c|}
\hline Variables & $\begin{array}{l}\text { Advice and } \\
\text { exercise }\end{array}$ & $\begin{array}{c}\text { Advice, exercise, } \\
\text { and true } \\
\text { acupuncture }\end{array}$ & $\begin{array}{c}P \text { value* } \\
\left(\mathrm{X}^{2} \text { test for trend }\right)\end{array}$ & $\begin{array}{l}\text { Advice, exercise, } \\
\text { and non-penetrating } \\
\text { acupuncture }\end{array}$ & $\begin{array}{c}\text { P value } \\
\dagger\left(x^{2} \text { test for trend }\right)\end{array}$ \\
\hline \multicolumn{6}{|l|}{ Six weeks: } \\
\hline Very satisfied & $47(45)$ & $62(55)$ & \multirow{5}{*}{0.07} & $71(62)$ & \multirow{5}{*}{0.008} \\
\hline Quite satisfied & $39(37)$ & 37 (33) & & $35(30)$ & \\
\hline No opinion & $10(10)$ & $8(7)$ & & $3(3)$ & \\
\hline Not very satisfied & $8(7)$ & $5(4)$ & & $6(5)$ & \\
\hline Not at all satisfied & $1(1)$ & $0(0)$ & & $0(0)$ & \\
\hline Missing data & 1 & 0 & & 0 & \\
\hline \multicolumn{6}{|l|}{ Six months: } \\
\hline Very satisfied & $32(31)$ & $38(35)$ & \multirow{5}{*}{0.5} & $56(50)$ & \multirow{5}{*}{0.006} \\
\hline Quite satisfied & $44(42)$ & $46(43)$ & & $39(35)$ & \\
\hline No opinion & $15(14)$ & $11(10)$ & & $6(5)$ & \\
\hline Not very satisfied & $10(10)$ & $11(10)$ & & $9(8)$ & \\
\hline Not at all satisfied & $3(3)$ & $2(2)$ & & $1(1)$ & \\
\hline Missing data & 1 & 1 & & 2 & \\
\hline \multicolumn{6}{|l|}{12 months: } \\
\hline Very satisfied & $28(29)$ & $33(33)$ & \multirow{5}{*}{0.2} & $47(44)$ & \multirow{5}{*}{0.009} \\
\hline Quite satisfied & $37(38)$ & $45(45)$ & & $40(38)$ & \\
\hline No opinion & $20(20)$ & $12(12)$ & & $7(7)$ & \\
\hline Not very satisfied & $8(8)$ & $9(9)$ & & $12(11)$ & \\
\hline Not at all satisfied & $5(5)$ & $2(2)$ & & $0(0)$ & \\
\hline Missing data & 1 & 0 & & 0 & \\
\hline
\end{tabular}

*Advice and exercise compared with advice, exercise, and true acupuncture.

†Advice and exercise compared with advice, exercise, and non-penetrating acupuncture. 


\section{WHAT IS ALREADY KNOWN ON THIS TOPIC}

Guidelines for the management of knee osteoarthritis emphasise patient education and exercise

Acupuncture alongside mainstream healthcare interventions has been advocated

\section{WHAT THIS STUDY ADDS}

Acupuncture did not provide greater reduction in pain scores when added to a course of advice and exercise delivered by physiotherapists

Benefits from acupuncture were limited to pain intensity and unpleasantness, were unlikely to be clinically significant, were mostly short lived, and could not be attributed to needling effects
Our trial failed to show that acupuncture is a useful adjunct to a course of individualised, exercise based physiotherapy for older adults with knee osteoarthritis. This adds to the existing body of evidence as acupuncture was compared with an effective comparison treatment.

\section{CONCLUSION}

Our trial addressed the three important questions recommended by the House of Lords report on complementary and alternative medicine in $2000 .{ }^{46}$ Firstly, true acupuncture did not show any greater therapeutic benefit than a credible control procedure in patients with a clinical diagnosis of knee osteoarthritis. Secondly, acupuncture was safe, with few, minor adverse events. Thirdly, acupuncture provided no additional improvement in pain scores compared with a course of six sessions of physiotherapy led advice and exercise. The small additional benefits from acupuncture were unlikely to be clinically significant, were limited to pain intensity and unpleasantness, were mostly short lived, and could not be attributed to specific acupuncture needling effects. Further research is needed to investigate the possible mechanisms of acupuncture, particularly the role of expectancy effects.

We thank Chan Vohora, Hannah Yates, Rhian Hughes, Jo Bailey, Gail White, Sue Weir, Claire Calverley, Wendy Clow, Peter Croft, and Krysia Dziedzic (Primary Care Musculoskeletal Research Centre); the physiotherapists based at the following NHS trusts: Amber Valley Primary Care Trust, South, Eastern, Heart of, and North Birmingham primary care trusts, East and South Staffordshire primary care trusts, Mid Staffordshire General Hospitals NHS Trust, East Cheshire Primary Care Trust, Mid Cheshire Hospitals NHS Trust, East Cheshire NHS Trust, Derby Hospitals NHS Foundation Trust, Worcestershire Acute Hospitals NHS Trust, Shrewsbury and Telford Hospital NHS Trust, Shropshire Community Primary Care Trust, Burton Hospitals NHS Trust, Robert Jones and Agnes Hunt Orthopaedic Hospital NHS Trust, South Warwickshire Primary Care Trust, South Warwickshire Hospitals NHS Trust, North Stoke, and Staffordshire Moorlands primary care trusts; and Adrian White, Michael Cummings, and Hugh MacPherson for categorising patients on the de qi sensation.

Contributors: All authors participated in the interpretation of the results and drafting of the article. NEF contributed to the design, funding application, advice and exercise intervention, training of the therapists, and management of the trial. ET contributed to the outcomes measured, data management, and analysis. PB contributed to the design, funding application, acupuncture interventions, and training of the therapists. JCH and EM contributed to the management of the trial, data collection, and training of the therapists. JY contributed to the recruitment procedures, data collection, and management. $\mathrm{EMH}$ contributed to the design, funding application, and management of the trial. NEF and EMH are guarantors.

Funding: This study was supported by a project grant from the Arthritis Research Campaign, UK (grant H0640) and Support for Science funding secured by the North Staffordshire Primary Care Research Consortium for NHS service support costs. NEF is funded by a primary care career scientist award from the Department of Health and NHS research and development, UK. $\mathrm{JCH}$ is funded by a lecturership in physiotherapy from the Arthritis Research Campaign, UK.

Competing interests: None declared.

Ethical approval: This study was approved by the West Midlands multicentre research ethics committee and by 13 local ethics committees. States $^{23}$ and Germany. ${ }^{24-26}$ We developed the acupuncture protocols to fit within current physiotherapy practice in the United Kingdom, however, and the protocols met the minimum criteria for adequacy of acupuncture.
1 Peat G, McCarney R, Croft P. Knee pain and osteoarthritis in older adults: a review of community burden and current use of primary health care. Ann Rheum Dis 2001;60:91-7. 
2 Duncan RC, Hay EM, Saklatvala J, Croft PR. Prevalence of radiographic osteoarthritis-it all depends on your point of view. Rheumatology 2006;45:757-60.

3 Cibere J, Trithart S, Koec JA, Thorne A, Zhang H, Singer J, et al. Pre-radiographic knee osteoarthritis is common in people with knee pain: results from a population-based study. Arthritis Rheum 2005;52(9 suppl):S509 (Abstract).

4 Primary Care Rheumatology Society. The management of osteoarthritis-guidelines. www.library.nhs.uk/musculoskeletal/ ViewResource.aspx?resID $=5317$.

5 Jordan KM, Arden NK, Doherty M, Bannwarth B, Bijlsma JWJ, Dieppe P, et al. EULAR recommendations 2003: an evidence based approach to the management of knee osteoarthritis: Report of a Task Force of the Standing Committee for International Clinical Studies Including Therapeutic Trials (ESCISIT). Ann Rheum Dis 2003;62:1145-55.

6 Roddy E, Zhang W, Doherty M, Arden NK, Barlow J, Birrell F, et al. Evidence-based recommendations for the role of exercise in the management of osteoarthritis of the hip or knee-the MOVE consensus. Rheumatology 2005;44:67-73.

7 Van Baar ME, Assendelft WJJ, Dekker J, Oostendorp RAB, Bijlsma JWJ. Effectiveness of exercise therapy in patients with osteoarthritis of the hip or knee. Arthritis Rheum 1999;42:1361-9.

8 Thomas KS, Miller P, Doherty M, Muir KR, Jones AC, O'Reilly SC. Cost-effectiveness of a two-year home exercise program for the treatment of knee pain. Arthritis Rheum 2005;53:388-94.

9 Hay EM, Foster NE, Thomas E, Peat G, Phelan M, Yates HE, et al. Pragmatic randomized clinical trial of the effectiveness of community physiotherapy and enhanced pharmacy review for knee pain in older people presenting to primary care. BMJ 2006;333:995-9.

10 Smidt N, de Vet HCW, Bouter LM, Dekker J. Effectiveness of exercise therapy: a best-evidence summary of systematic reviews. Aust Physiother 2005;51:71-85.

11 Oxford Pain Site. NSAIDS and adverse effects. Bandolier. www.jr2.ox. ac.uk/bandolier/booth/painpag/nsae/nsae.html.

12 Pound P, Britten N, Morgan M, Yardley L, Pope C, Daker-White G, et al. Resisting medicines: a synthesis of qualitative studies of medicine taking. Soc Sci Med 2005;61:133-55.

13 Arthritis Care. Osteoarthritis nation: the most comprehensive UK report of people with osteoarthritis. London: Arthritis Care, 2004.

14 Breivik H, Collett B, Ventafridda V, Cohen R, Gallacher D. Survey of chronic pain in Europe: prevalence, impact on daily life and treatment. Eur J Pain 2006;10:287-333.

15 Vickers A. Complementary medicine. BMJ 2000;321:683-6.

16 Thomas KJ, Nicholl JP, Fall M. Access to complementary medicine via general practice. BrJ Gen Pract 2001;51:25-30.

17 NIH Consensus Conference. Acupuncture. JAMA 1998;280:1518-24.

18 White AR, Filshie J, Cummings TM. Clinical trials of acupuncture: consensus recommendations for optimal treatment, sham controls and blinding. Complement Ther Med 2001;9:237-45.

19 Derry CJ, Derry S, McQuay HJ, Moore RA. Systematic review of systematic reviews of acupuncture published 1996-2005. Clin Med 2006;6:381-6.

20 Ezzo J, Hadhazy V, Birch S, Lao L, Kaplan G, Hochberg M, et al. Acupuncture for osteoarthritis of the knee: a systematic review. Arthritis Rheum 2001;44:819-25.

21 White AR, Foster NE, Cumming M, Barlas P. Acupuncture for chronic knee pain: a systematic review. Rheumatology 2007;46:384-90.

22 Vas J, Mendez C, Perea-Milla E, Vega E, Panadero MD, Leon JM, et al. Acupuncture as a complementary therapy to the pharmacological treatment of osteoarthritis of the knee: randomized controlled trial. BMJ 2004;329:1216-21.

23 Berman BM, Lao L, Langenberg P, Lee WL, Gilpin AMK, Hochberg MC. Effectiveness of acupuncture as adjunctive therapy in osteoarthritis of the knee: a randomized, controlled trial. Ann Intern Med 2004;141:901-10.

24 Witt C, Brinkhaus B, Jena S, Linde K, Streng A, Wagenpfeil S, et al. Acupuncture in patients with osteoarthritis of the knee: a randomized trial. Lancet 2005;366:136-43.
25 Scharf HP, Mansmann U, Streitberger K, Witte S, Kramer J, Maier C, et al. Acupuncture and knee osteoarthritis: a three-armed randomized trial. Ann Intern Med 2006;145:12-20.

26 Witt CM, Jena S, Brinkhaus B, Liecker B, Wegscheider K, Willich SN. Acupuncture in patients with osteoarthritis of the knee or hip: a randomized, controlled trial with an additional nonrandomised arm. Arthritis Rheum 2006;54:3485-93.

27 Hay E, Barlas P, Foster N, Hill J, Thomas E, Young J. Is acupuncture a useful adjunct to physiotherapy for older adults with knee pain? The "Acupuncture, Physiotherapy and Exercise" (APEX) study. BMC Musculoskelet Disord 2004;5:31.

28 Streitberger K, Kleinhenz J. Introducing a placebo needle into acupuncture research. Lancet 1998;352:364-5.

29 Foster NE, Barlas P, Dziedzic K, Daniels J, Gray R. Current physiotherapy management of knee osteoarthritis informs a clinical trial [abstract]. Rheumatology 2000;39(suppl 1):166.

30 Foster NE, Barlas P, Daniels ], Dziedzic K, Gray R. Use of acupuncture by physiotherapists in the treatment of osteoarthritis of the knee: current trends inform a clinical trial. Birmingham, UK: Proceedings of the Chartered Society of Physiotherapy Congress, Oct 1999;27.

31 Kleinhenz J, Streitberger K, Windeler J, Güssbacher A, Mavridis G, Martin E. Randomised clinical trial comparing the effects of acupuncture and a newly designed placebo needle in rotator cuff tendinitis. Pain 1999;83:235-41.

32 Bellamy N. WOMAC osteoarthritis index. A user's guide. Ontario: University of Western Ontario, 1996.

33 Jinks C, Jordan K, Croft P. Measuring the population impact of knee pain and disability with the Western Ontario and McMaster Universities osteoarthritis index (WOMAC). Pain 2002;100:55-64.

34 Pham T, Van der Heijde D, Lassere M, Altman RD, Anderson JJ, Bellamy N, et al. Outcome variables for osteoarthritis clinical trials: the OMERACT-OARSI set of responders criteria. J Rheumatol 2003;30:1648-54

35 Pham T, Van der Heijde D, Altman RD, Anderson JJ, Bellamy N, Hochberg M, et al. OMERACT-OARSI initiative: Osteoarthritis Research Society International set of responder criteria for osteoarthritis clinical trials revisited. Osteo Cartilage 2004:12:389-99.

36 Vincent CA, Lewith G. Placebo controls for acupuncture studies. J R Soc Med 1995;88:199-202.

37 O'Reilly SC, Muir KR, Doherty M. Effectiveness of home exercise on pain and disability from osteoarthritis of the knee: a randomised controlled trial. Ann Rheum Dis 1999;58:15-9.

38 Norman GR, Streiner DL. Biostatistics: the bare essentials. St Louis: Mosby, 1994:160.

39 Office for National Statistics. Standard occupational classification 2000, vol. 2. The coding index. London: Stationery Office, 2000:4.

40 Office for National Statistics. The national statistics socio-economic classification user manual. Version 1. ONS: London, 2002:4

41 Berman BM, Singh BB, Lao L, Langenberg P, Li H, Hadhazy V, et al. $A$ randomized trial of acupuncture as an adjunctive therapy in osteoarthritis of the knee. Rheumatology 1999;38:346-54

42 Campbell A. Point specificity of acupuncture in the light of recent clinical and imaging studies. Acupunct Med 2006;24:118-22.

43 Lund I, Lundeberg T. Are minimal, superficial or sham acupuncture procedures acceptable as inert placebo controls? Acupunct Med 2006;24:13-5.

44 Bausell RB, Lao L, Bergman S, Berman BM. Is acupuncture analgesia an expectancy effect? Preliminary evidence based on participants' perceived assignments in two placebo-controlled trials. Eval Health Prof 2005;28:9-26.

45 Pariente J, White P, Frackowiak RS, Lewith G. Expectancy and belief modulate the neuronal substrates of pain treated by acupuncture. Neuroimage 2005;25:1161-7.

46 House of Lords Science and Technology Committee. Sixth report: complementary and alternative medicine. London: Stationery Office, 2000. www.parliament.thestationeryoffice.co.uk/pa/ld199900/ Idselect/ldsctech/123/12301.htm.

Accepted: 8 July 2007 